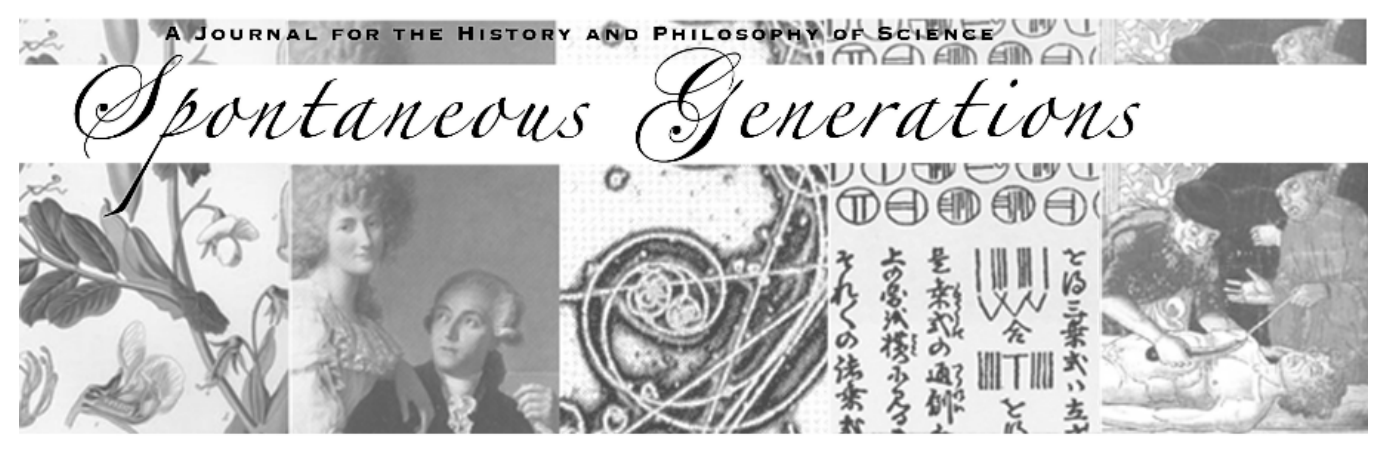

\title{
The Relevance of Scientific Practice to the Problem of Coordination
}

\section{Author(s): Andrew Peterson}

Source: Spontaneous Generations: A Journal for the History and Philosophy of Science, Vol. 5, No. 1 (2011) 44-57.

Published by: The University of Toronto

DOI: $\underline{10.4245 / \text { sponge.v5i1.14607 }}$

\section{EDITORIAL OFFICES}

Institute for the History and Philosophy of Science and Technology

Room 316 Victoria College, 91 Charles Street West

Toronto, Ontario, Canada M5S 1K7

hapsat.society@utoronto.ca

Published online at jps.library.utoronto.ca/index.php/SpontaneousGenerations ISSN 19130465

Founded in 2006, Spontaneous Generations is an online academic journal published by graduate students at the Institute for the History and Philosophy of Science and Technology, University of Toronto. There is no subscription or membership fee. Spontaneous Generations provides immediate open access to its content on the principle that making research freely available to the public supports a greater global exchange of knowledge. 


\title{
Peer-Reviewed
}

\section{The Relevance of Scientific Practice to The Problem of Coordination*, $\dagger$}

\begin{abstract}
Andrew Peterson
In his early work on the problem of coordination, Hans Reichenbach introduced axioms of coordination to describe the relationship between theory and observation. His insistence that these axioms are determinable a priori, however, causes him to ignore the normative dimensions of scientific inquiry and, in turn, generates a misleading interpretation of the theory-observation relationship. In response, I propose an alternative approach that describes this relationship through the framework of scientific practices. My argument will draw on two examples that have not been explored by the philosophical literature in the context of coordination problems: the clinical definition of death and Stanley Prusiner's prion hypothesis.
\end{abstract}

\section{The Problem With Reichenbach's Solution to The Problem of COORDINATION}

In his early discussion of the relationship between geometry and physics, Hans Reichenbach introduced axioms of coordination to describe the linkage between scientific theory and concrete observation. In his mind, the highly abstract expressions of theory were clearly distinguishable from the physical observations they came to describe, yet the means by which they became related remained entirely unclear. Responding to Kant's synthetic a priori and in an effort to understand the philosophical foundations of relativity theory, Reichenbach argued that theory and observation are linked through axioms of coordination stipulated by the scientist prior to empirical application. Such axioms were believed to establish the a priori grounds upon which the use of a theoretical expression became empirically meaningful. The theoretical expression, "the meter," for example, becomes empirically meaningful only after its a priori coordination to a physical object in Paris has been established (Reichenbach 1958, 14-15).

* I am greatly appreciative of the valuable comments provided by Isabelle Peschard, Bas van Fraassen, Kris Kemtrup, and Tyrus Fisher on multiple drafts of this paper.

$\dagger$ Received 7 December 2010. Accepted 28 July 2011.

$\ddagger$ Andrew Peterson's work focuses on the philosophy of medical science and bioethics in clinical and research settings. He has special interest in neurological conditions and reintegration challenges faced by combat veterans. Andrew Peterson received his MA from San Francisco State University, is a PhD candidate at The University of Western Ontario, and is a member of the Rotman Institute of Philosophy.

Spontaneous Generations 5:1 (2011) ISSN 1913-0465. University of Toronto. 
Contrary to Reichenbach's position, I argue that the interpretive framework of axioms of coordination obscures the importance of scientific practices in the coordination process. Through his insistence that coordinative axioms are determinable a priori, Reichenbach presumes that the theory-observation relationship is initially intelligible independent of the concrete scientific practices that constitute the involved, experiential aspects of scientific inquiry. On my view, the linkage between theory and observation is not determinable a priori. Rather, this linkage is contingent upon the normative constraints that emerge from the involved, practical activities of a scientific culture.

To defend this claim, I exhibit the deficiencies of Reichenbach's position by applying it broadly to two examples previously unexplored by the philosophical literature in the context of coordination problems. First, I explore the clinical definition of death and its coordination to physical signs manifested in a patient's body. I show that Reichenbach's position does not account for the legal norms that constrain such medical diagnoses in practice. I then turn to a second example, Stanley Prusiner's prion hypothesis, to defend Reichenbach's position by removing the normative dimension of coordination in the case study. I show, however, that despite such an alteration to the example, the prion hypothesis is also contingent upon social norms and a value-laden experimental culture. On the basis of these criticisms, I argue that axioms of coordination are insufficient for explaining the relationship between theory and observation when science is understood in terms of its practices.

\section{Reichenbach's A Priori Axioms of Coordination.}

Beginning as early as his 1915 doctoral dissertation, Reichenbach devoted himself to answering the following question: how do the objects of knowledge in mathematics link up with the objects of knowledge in physics? (cf. Reichenbach 1965 , 34) The epistemic strength of mathematical theorems is determined by internal coherence, yet "the truth of physical propositions...depends on relations to something external" (Reichenbach 1965, 34). In light of this, he considered how concrete observations correlate with a theoretical expression such as $P V=r T$. Though the theoretical expression is logically coherent in and of itself, its connection to the empirical manifestations of "pressure," "volume," and "temperature" is altogether vague. "What is lacking," Reichenbach claimed, "is...the assertion that the system of equations is true for reality" (Reichenbach 1965, 36).

A priori axioms of coordination were introduced as the key for clarifying this vague relationship. As previously described, a theoretical expression such as "the meter" is coordinated with the physical object in Paris in an effort to render a theory that is empirically meaningful prior to application in physical measurement (cf. Reichenbach 1958, 14-15). Another example discussed by Reichenbach involved the coordination of geometric concepts to the physical manifestation of the earth. 
"By calling the earth a sphere, we are coordinating the mathematical figure of a sphere to certain visual and tactile perceptions that we call 'perceptual images of the earth" (Reichenbach 1965, 37). These axioms of coordination, stipulated prior to experience, not only establish a correlation between seemingly discrete components of scientific inquiry-theory and observation-they also establish the grounds for believing that abstract theory is expressing something of empirical importance.

Reichenbach observed, however, that the correlation these coordinations provide is far more "peculiar" than such simple examples might lead us to believe (Reichenbach 1965, 37). Unlike internal coordinations amongst mathematical equations, the definition of theoretical sets in relation to an observational side of the coordination is intrinsically problematic. Reichenbach described the problem in the following way:

For example, if two sets of points are given, we establish a correspondence between them by coordinating to every point of one set a point of the other set. For this purpose, the elements of each set must be defined; that is, for each set there must exist another definition in addition to that which determines the co-ordination to the other set. (Reichenbach 1965, 37)

But, as Reichenbach observed, "such definitions are lacking on the side of the coordination dealing with the cognition of reality" (Reichenbach 1965, 37). In response to this paradox, Reichenbach argues: "although the equations, that is, the conceptual side of the coordination, are uniquely defined, the 'real' is not. On the contrary, the 'real,' is defined by the coordinations to the equations" (Reichenbach 1965, 37-38). In effect, the observational side of the coordination is initially subsumed under the defining power of the theory as established through the axioms. The perceivable object in Paris, in other words, is defined by its coordination to the mathematic theory expressed through the term "the meter" and not the other way around.

Anticipating the criticism that this description undermines the importance of the observable side of the coordination, Reichenbach added, "although there is a coordination to undefined elements, it is restricted, not arbitrary" (Reichenbach 1965, 42). This restriction is imparted by observable correlates derived from reality. Though perception does not supply the definition of what is real (cf. Reichenbach 1965, 42), the physical side of the coordination constrains the scope of concepts intelligibly coordinated with it. If this were not the case, one could easily presume that any set of physical correlates is "fictitious, a hypothesized thing-in-itself" (Reichenbach 1965, 42).

In the overall context of Reichenbach's description of a priori axioms, this "mutuality of coordination" (Reichenbach 1965, 42) is perhaps the most puzzling. Throughout he insisted that the axioms of coordination are determinable prior 
to experience, yet the claim that both sides of the coordination are mutual leads one to believe the coordinated relationship is far more complex. Moreover, Reichenbach's latter introduction of "axioms of connection," as detailed by Friedman (1999) and Padovani (2009), opens the possibility of a relativized a priori. These assertions, I believe, come very close to appreciating the importance of concrete scientific practices within the coordination procedure. Yet Reichenbach's emphasis on the axiom's initial reliance on a prioricity, in addition to his inadequate treatment of the experimental dimensions of scientific inquiry, ultimately leaves us with the impression that this mutuality is rather asymmetrical.

This truncated exegesis of Reichenbach's position leaves us with four principles:

1. Theory and object are linked together through a priori axioms of coordination.

2. Axioms of coordination are stipulated prior to empirical application.

3. The physical side is defined by its coordination to a theory.

4. There is mutuality to the coordination; coordinations are not arbitrary. ${ }^{1}$

The question now remains: Are Reichenbach's axioms an accurate framework for interpreting the relation between theory and observation as it manifests itself within the context of concrete, historically situated scientific practices? Considering that Reichenbach's emphasis on a prioricity as an initial function of coordination leaves no room for the normative dimensions of the theory, observation relationship, I hope to show that it is not. Only a position that grounds its interpretation of this relationship in the common currency of scientific practices will coherently appreciate the normative constraints that arbitrate coordinations within a broad range of scientific fields. Reichenbach's a priori axioms of coordination, on my view, cannot do this work for us.

\section{Are There Coordination Problems in The Life Sciences?}

Consider the problem of coordination applied to the following scenario:

A 35-year-old male recently involved in a car accident has been admitted to the San Francisco General Hospital for surgical treatment of a traumatic brain injury. During an operation to relieve cranial pressure, the patient's vital stats begin to decline. He becomes hypotensive, his pulse oxygenation drops below 60\%, and his ECG becomes erratic. Despite the best efforts of the anesthesiologist

1 As I have mentioned earlier, this fourth component of Reichenbach's axioms appears out of place. I will take this up in greater detail in a later portion of this paper. 
to stabilize the patient, in addition to a prolonged ceremony of resuscitative techniques, he arrests and the anesthetic monitoring equipment falls silent. Reluctantly, the attending surgeon removes her cap and mask and in a fit of self-deprecation makes the call, "time of death, 23:00 hours."

In relation to Reichenbach's position, this example is not philosophically trivial. Though we have replaced geometric concepts with a clinical definition, the fundamental coordination problem still remains: how does the medical scientist link the theoretical definition of death to a set of physical correlates manifested in a patient's body? How does "death" as a clinical definition become empirical?

\section{The Two Dimensions of Coordination in Medical Practice}

Because coordination problems are apparent in scientific fields beyond physics, how might we articulate a plausible understanding of the theory, observation relationship that is applicable to a broad range of scientific inquiry-including the life sciences? To answer this, consider the time-honored medical apothegm, "a person is dead when a physician says so" (Iserson 1994, 19). In the case of coordination within medical practice, what makes this adage true? Is it on the grounds of an initial a priori axiom of coordination? Or some other dimension of scientific practice unaccounted for by Reichenbach's analysis? To make sense of this I believe it is useful to distinguish two dimensions of coordinated relationships. First, what I call the epistemological dimension, strictly concerns the coordination of theory to natural phenomena as expressed through the accepted theoretical criteria and measurement procedures. This epistemological dimension of clinical death is determinable, in part, through a Reichenbachian framework. One may, for example, cross-reference this medical scenario with the truncated coordination principles previously described: $(1,2)$ Medical scientists coordinate the theoretical definition with a set of observations prior to pronouncing the patient "dead." (3) The physical manifestations of death as recorded by the measurement procedures are defined in relation to the theoretical criteria of death (lack of certain electroencephalographic activity, for example, is a relevant empirical manifestation relative to the established theoretical criteria of lower or higher order brain death). And (4) the physical correlates indicated by observable vital signs constrain the physician's ability to stipulate "death" arbitrarily.

The second and significantly more important dimension, what I call the normative dimension, is the set of legal and social conditions under which a physician can stipulate the diagnosis whether or not the components of the epistemological dimension obtain. Though the epistemological dimension is a necessary condition of coordination, it is ultimately insufficient for coordinating the clinical definition of "death" in medical practice. A person is certainly dead 
when the physician says so, but only insofar as the epistemological dimension of the coordination agrees with social and legal norms in which it is situated. By virtue of Reichenbach's emphasis on the initial a prioricity of coordinative axioms, he fails to acknowledge the normative constraints that dictate which coordinations are legally or morally permissible regardless of their epistemological merit.

\section{The Reciprocal Interplay of These Dimensions Throughout Medical HISTORY}

Evidence for this tension between the normative and epistemological dimensions has been appreciable ever since medical scientists began to level the diagnosis of "death" in a clinical setting. Prior to the sixteenth century, for example, the heretical nature of autopsies stifled the growth of medical knowledge and limited the established theoretical criteria to the nascent cardiopulmonary descriptions of Aristotle, Galen, and Praxagoras of Kos (cf. Nuland, 2003). William Harvey's seventeenth century research of the circulatory system, in addition to Rene Laennec's serendipitous invention of the stethoscope slowly altered these criteria by providing a more predictable and epistemologically coherent account of "death's" empirical manifestations (cf. Harvey 1878; cf. Whetstine 2008). Despite this, growing cultural anxieties about being buried alive (cf. Whetstine 2008), in addition to new resuscitation techniques of the eighteenth and nineteenth centuries, hastened another reformulation of the theoretical criteria. "Death" was not only the cessation of particular physiological systems, but also its irreversibility. In the most recent example of this tension, the development of the electroencephalograph (EEG), in addition to advanced neurological imaging techniques (cf. Plum 1999) predicated the legislative response of "whole brain" and "higher brain" theories of "death". ${ }^{2}$ This distinction was established to arbitrate the tenuous diagnoses of comatose patients who exhibited empirical manifestations consistent with the cardiopulmonary criteria, yet were kept "alive" through newly developed life sustaining technologies ${ }^{3}$ (cf. President's Commission 1981).

In light of such historical evidence, it is clear that physicians have not been free to stipulate a priori axioms of coordination between the clinical definition of death and a set of empirical manifestations without being deferential to the normative constraints of their time. Not only is it evident that the epistemological dimensions of the coordination have changed in relation to the theoretical and technological advancements, it is also clear that normative pressures frequently

2 In fact, recent neuro-imaging studies through both The University of Western Ontario's Center for Brain and Mind and Cambridge, document conscious, responsive brain activity of patients in persistent vegetative states, thus further altering the relevant diagnostic criteria of certain neurological disorders including brain death.

3 Artificial respiration, hydration, and nutrition for example. 
intervened to arbitrate the course of medical research by dictating what counts as legally or morally permissible coordinations regardless of scientific precedent. A paradigmatic example of this is the most recent distinctions of "brain death" established to placate the normative concerns of declaring death prematurely for the sake of harvesting transplant organs. Though the epistemological dimensions of the diagnosis may warrant the stipulation of "death" in the clinical setting, the coordination will ultimately be overridden by social norms that restrict premature, and thus unjust "death" from occurring. Because Reichenbach's position focuses narrowly on the initial a prioricity of coordinations, his position is insufficient for explaining this normative dimension of the theory, observation relationship emerging from culturally embedded scientific practices.

Any articulation of the relationship between theory and observation in the life sciences, therefore, must provide an interpretive framework for understanding the interplay between the epistemological and normative dimensions of coordination. Death, as a clinical diagnosis, is made empirical partly on the basis of epistemological criteria; after all, the natural phenomena we associate with clinical definition of death are real and can be measured. But the stipulation of this clinical definition as an empirical diagnosis is ultimately arbitrated by the normative conditions that emerge out of the practice of medicine itself. As I see it, Reichenbach's axioms of coordination fail to acknowledge this very important aspect of the theory, observation relationship.

\section{Vi. Are Axioms of Coordination Still Effective If We Ignore The Normative Dimension?}

To be charitable to Reichenbach, we should presume that he did not have these normative dimensions in mind when laying the groundwork for axioms of coordination. One could reasonably insist that in spite of his failure to acknowledge normativity, axioms of coordination are still useful for understanding the purely epistemological relationship between concept and object evident in all fields of scientific inquiry. Assuming this is true, is the success of Reichenbach's a priori axioms of coordination preserved when judged solely on their epistemological dimension? In other words, if Reichenbach's framework were applied to scientific fields with less normative influence, will his argument be successfully rendered? One might initially think so, for Reichenbach developed his position in response to the changing theoretical landscape of physics in the mid-twentieth century, not the morally charged practice of medicine. But to claim the application of his position to coordination problems across a broad spectrum of scientific inquiry is, perhaps, insensitive to the target of his research presumes that physics is somehow immune to the problems that scientific practices present. Thus, if it can be shown that the epistemological dimension of coordination is grounded in the practices of science, and that these practical activities are always and already value-laden regardless of scientific field, special treatment 
of Reichenbach's position will be unwarranted since it excludes the influence of scientific practices all empirical sciences, by definition, share.

To show this, take as an example this isolated coordination stipulated by Stanley Prusiner during his 1980s laboratory research of the enigmatic pathogen Scrapie:

[...] the molecular properties of the scrapie agent differ from those of viruses...Its resistance to procedures that attack nucleic acids, its resistance to inactivation by heat, and its apparent small size all suggest that the scrapie agent is a novel infectious entity. [...] In place of such terms as 'unconventional virus' or 'unusually slow virus like agent', the term 'prion' is suggested. Prions are small proteinasceous infectious particles which are resistant to inactivation by most procedures that modify nucleic acids. The term 'prion' underscores the requirement of a protein for infection. (Prusiner 1982, 141, my emphasis)

This example is certainly in accordance with the initial stages of Reichenbach's coordination procedure. Like the aforementioned example of clinical death, Prusiner's stipulative coordination between the theoretical expression "prion" and a set of physical correlates can be mapped with the truncated version of Reichenbach's principles outlined above. $(1,2)$ A scientist stipulated the axiom prior to empirical investigation by coordinating the theoretical expression "prion" with a set of physical correlates; (3) the physical manifestations of the infectious agent are defined by their relation to the theoretical framework expressed by the term "prion"; and (4) the physical manifestations of the pathogen constrains its coordination to a strict subset of theoretical frameworks.

Notwithstanding this agreement, there is still an important element absent from this coordination. I anticipate, for example, that those unfamiliar with Stanley Prusiner's research will be puzzled for lack of contextual information surrounding this citation. The reason for such confusion, I believe, is that the epistemological dimension of coordination is only intelligible in terms of its relation to the concomitant experimental network in which it is situated. In other words, the revolutionary impact of Prusiner's prion hypothesis is only intelligible on the experimental background of virology and molecular biology during the late twentieth century. Though Prusiner's prion hypothesis does not entail the same normative constraints as the clinical definition of death, the coordination as construed under Reichenbach's framework still does not escape the concrete, historically situated experimental culture in which it is established. 


\section{Vil. Experimental Networks and The Epistemological Dimension of} COORDINATION

To make this assertion more robust, consider a brief sketch of the experimental network that lent itself to Prusiner's "prion" coordination: Early twentieth-century research into a neurodegenerative disease named Kuru, a condition endemic to the native Fore people of the Papua New Guinea highlands, set in motion a network of experimental inquiry that ultimately culminated in Prusiner's research (cf. Anderson 2008). The newly christened theoretical constraints of molecular biology, particularly Crick's "Central Dogma" of protein synthesis, ${ }^{4}$ led many to believe that Kuru, and the similar neuropathy of sheep and goats, Scrapie, were viral in constitution. Concurrent veterinary studies, however, attempted to destroy the agent's genetic material through exposure to high temperatures (Stamp 1962), formalin (Pattison 1965), and ultra-violet radiation (Alper et al. 1966)-procedures known to inhibit viral proliferation-yet they were all shown to be unsuccessful. Prusiner, having observed this experimental network unfold, started from the radically different assumption that the pathogens were something other than a virus. Instead of attempting to inhibit genetic material, he exposed Scrapie to

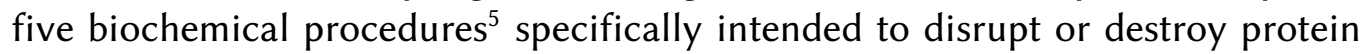
structure (Prusiner et al. 1981, 6677). The results of these tests showed, contrary to Crick's "Central Dogma”, that the scrapie agent was inhibited after destroying its proteinasceous component rather than its nucleic acids (cf. Prusiner et al. 1981). The aforementioned citation was published in 1982 shortly after Prusiner conducted these particular experimental investigations.

It is easy to see that Prusiner's stipulation of the relationship between the theoretical expression "prion" and a set of physical correlates was mediated through a rich and dynamic experimental network. Prusiner's coordination is certainly devoid of the overt normativity observed in the diagnosis of clinical death, but his coordination was still constrained by the tacit expectations of a concrete, historically situated scientific culture. In the midst of scientific inquiry, a scientist must make decisions as to what factors are epistemologically relevant for a particular coordination. This decision of relevance, however, is not determinable a priori as Reichenbach's coordinative axioms suggest. Nor are they determinable from the empirical side of the coordination-what his latter "coordinations of connection" were presumably intended to explain (cf. Friedman 1999, 61). On the

${ }^{4}$ Crick's "Central Dogma" laid the conceptual groundwork for unidirectional protein synthesis. The transfer of genetic information through DNA transcription and RNA translation into protein structure was taken as one way; "once [genetic] 'information' has passed into a protein it cannot get out again" (Crick 1958, 153, my gloss in brackets). In effect, Crick's "Dogma" forbade the transfer of genetic information from protein to protein-or from protein back to nucleic acid. Genetic material was necessary for the replication of any living organism.

5 These tests exposed Scrapie to proteinase K, diethylprocarbonate, sodium dodecyl sulfate, chaotropic ions, and phenol-all biochemical agents with known protein inhibiting properties. 
contrary, it is only by situating these coordination decisions within a network of practically involved scientific activities-a dynamic experimental culture-that relevant factors become transparent. Prusiner, for example, made the decision to coordinate one set of observable correlates with "prion" as opposed to another based on the expectations of the experimental culture in which he, as a scientist, was situated. The relevant factors of his decision are unintelligible outside the context of these concrete scientific activities.

As long as coordinations are initially taken as a priori axioms, in isolation from a concrete experimental network, there is no way to articulate why certain coordinations are deemed more relevant than others. In effect, Reichenbach's position takes a retrospective approach to coordinations where it is presumed that the relation between theory and observation couldn't have been any other way (cf. van Fraassen 2008, 122). Yet this is contradictory to the state of affairs faced by a scientist in the midst of a value-laden experimental network. ${ }^{6}$ Because the common currency of scientific practices exhibited by all experimental sciences continues to constrain the epistemological dimension of coordination, Reichenbach's axioms fail on this count as well.

\section{Vili. An Alternative Approach to Coordination Problems}

While Reichenbach insists that a priori axioms of coordination are necessary for determining the relationship between concept and object, it is my contention that these two aspects of scientific inquiry are always and already inextricably linked. Because the relationship is manifested in both an epistemological and normative dimension, it is evident that coordinations evolve out of dynamic, value-laden networks of concrete experimental inquiry. If the starting point of the theory, observation relationship is framed this way, the normative influences of scientific activity are taken into consideration and the problems generated by Reichenbach's a priori emphasis are dissolved. In short, by revising the assumption that coordinations are initially independent from the concrete, historically situated activities of science, we can adequately answer the questions Reichenbach set out to solve.

Though Hans-Jörg Rheinberger (1997) does not respond directly to Reichenbach, his description of experimental investigation is a useful point of departure for critiquing axioms of coordination. Rheinberger, like Reichenbach, certainly appreciates the importance of the theory-observation dichotomy implicit within all scientific investigation. But he diverges from Reichenbach in arguing that this relationship is generated through a highly dynamic, amorphous "experimental system." This, he believes, supplants the received view that

${ }^{6}$ Rheinberger makes the similar observation, "No historiography of science can escape this movement of recurrence implanted into its very subject matter... For every new scientific object sheds a 'recurrent light' on those by which it was preceded" (Rheinberger 1997, 33). 
construes experimental inquiries as isolated empirical instances. "A researcher," he claims, "does not, as a rule, deal with isolated experiments in relation to a theory, but rather with a whole experimental arrangement designed to produce knowledge that is not yet at his disposal" (Rheinberger 1997, 27). From this dynamic experimental system, Rheinberger asserts that "the phenomena or material entities and the concepts they come to embody" are inextricably co-generated (Rheinberger 1997, 27). "Practice and concepts thus 'come packaged together" (Rheinberger 1997, 27). As expressed here, theory and observation are co-emergent from the historically situated practices of experimental inquiry rather than in need of coordination through a priori axioms. Though Reichenbach argues coordination is necessary to coherently link these discrete categories, Rheinberger insists they are always and already reciprocally interwoven.

The "mutuality" (Reichenbach 1965, 42) of coordination previously mentioned in principle (4) of Reichenbach's axioms is, perhaps, the closest his position comes to appreciating the nuances of Rheinberger's description. To claim that coordinations are not arbitrary because the empirical constrains the theoretical is to agree that the relationship between theory and observation is, in fact, reciprocal. Moreover, axioms of connection, "empirical laws in the usual sense involving terms and concepts that are already sufficiently well defined" (Friedman $1999,61)$ indicates that Reichenbach took this concern seriously when organizing his philosophical response to Kant, Newtonian laws, Euclidian geometry, and Galilean kinematics (cf. Friedman 1999, 59-62; Padovani 2009). But why, then, does Reichenbach insist that the starting point of coordinations is grounded in an a priori procedure? Why, for example, does he claim that 'the 'real,' is defined by the coordinations to the equations" (Reichenbach 1965, 37-38) and not the other way around? Even the subtle change to coordinative definitions (cf. Reichenbach 1958) which gives rise to the relativized a priori still does not reflect the importance of the physical side of the coordination as this initial gesture toward "mutuality" suggests. Because of Reichenbach's thoroughgoing emphasis on the initial a prioricity of coordinations, in addition to his silence toward the culturally embedded practices of science, this claim appears drastically out of place.

\section{Examples of Theory, Observation Dynamism}

To see that this dynamic relationship between theory and observation has significant explanatory power, consider again Prusiner's prion research under the organizing framework of an experimental system. Prusiner certainly stipulated the "prion" coordination as Reichenbach would describe, yet he did so upon the background of a value-laden experimental culture that ultimately rendered his assertion intelligible. The techniques of virology, veterinary epidemiology, and nascent molecular biological theory all added to the growing body of knowledge that constituted the concrete experimental network from which the coordination 
emerged. The coordination that typifies the expression "prion" was not stipulated over and above the observations of Prusiner's five biochemical tests. Rather it was generated from practical scientific activities that were always and already imbued with the norms of a situated scientific culture.

The explanatory power of an experimental system can likewise be applied to the clinical definition of death and the respective normative constraints that guide a physician's diagnosis. Innovations throughout the history of medical science have called for frequent revisions to the theory-observation coordination of the clinical criteria of death. This co-emergence of medical concept and clinical object, in turn, hastened the necessity of normative arbitration for borderline cases where diagnosis was entirely unclear. We saw previously that this alteration of coordinations poses significant problems for Reichenbach's a priori axioms, particularly because the stipulation of "death" as a clinical diagnosis evolved in relation to the normative constraints of medical practice. But taken under the framework of an experimental system, this co-evolution of the normative and epistemological dimensions of death as a clinical diagnosis is an entirely intelligible phenomenon. Concept and object co-evolved from the practical, value-laden activities of biomedical science.

\section{Lessons To Be Learned}

An interpretation of the theory-observation relationship that is grounded in concrete and historically situated scientific practices succeeds at accounting for the normative dimensions of coordination that Reichenbach's axioms do not. Reichenbach certainly identified the salient coordination problems shared by all regions of scientific inquiry, yet his solution ultimately fails by not accounting for the common currency of practically involved experimental activities. Whether we speak of medicine, molecular biology, or physics, we are still laying claim to an empirical investigation that is conducted by value-laden cultures at a particular time and place. The concrete activities of science are, therefore, never immune to normative constrains and any articulation of coordinations must take into consideration such influential factors.

By accepting a position that construes theory as inextricably linked with observation and thus emerging from a historically situated network of scientific practices, we gain insight into the important normative dimension of the theory, observation relationship. Rather than construing coordinations as determinable independent of scientific activity, the theory, observation relationship should be grounded in the value-laden practices of science. I have mentioned two examples from biomedicine to show that normativity plays a significant role within coordination. But this does not exclude the potential of applying this analysis to other areas of scientific inquiry where the relationship between 
concept and object is at issue. ${ }^{7}$ For all regions of scientific inquiry that are subject to coordination problems share the common feature of their practices, and a sufficient answer to the problem of coordination must take this common currency as its principle consideration.

\author{
Andrew Peterson \\ Department of Philosophy \\ The University of Western Ontario \\ 1151 Richmond Street, Stevenson Hall \\ London Ontario, Canada, N6A 3K7 \\ apeter62@uwo.ca
}

\title{
REFERENCES
}

Alper, Tikvah, D.A. Haig, and M.C> Clarke. 1966. The Exceptionally Small Size of the Scrapie Agent. Biochemical and Biophysical Research Communications vol. 22 no. 3: 278-84. doi:10.1016/0006-291X(66)90478-5

Anderson, Warwick. 2008. The Collectors of Lost Souls: Turning Kuru Scientists Into Whitemen. Baltimore, MD: John Hopkins University Press.

Crick, Francis. 1958 On Protein Synthesis. Symposia of the Society of Experimental Biology no. 12: 138-63.

Friedman, Michael. 1999. Reconsidering Logical Positivism. Cambridge, UK: Cambridge University Press.

Iserson, Kenneth. 2001. Death to Dust What Happens to Dead Bodies? Second Edition. Tucson, AZ: Galen Press.

Nuland, Sherwin. 2003. The Doctor's Plague. New York, NY: Norton and Company.

Padovani, Flavia. 2011. Relativizing the Relativized A Priori: Reichenbach's Axioms of Coordination Divided. Synthese vol. 181 no. 1: 41-62. doi:10.1007/s11229-009-9590-0

Pattison, Iain H. 1965. Resistance of The Scrapie Agent to Formalin, Journal of Comparative Pathology vol. 75 no. 2: 159-64. doi:10.1016/0021-9975(65)90006-X

Pernick, Martin. 1988. Back From the Grave: Recurring Controversies Over Defining and Diagnosing Death in History. In Death: Beyond the Whole-Brain Criteria, ed. Richard Zaner. 17-74. Norwell, MA: Kluwer.

Plum, Fred. 1999. Clinical Standards and Technological Confirmatory Tests in Diagnosing Brain Death. In The Definition of Death: Contemporary Controversies, eds. Stuart Younger, Robert Arnold, Renie Schapiro, 34-66. Baltimore, MA: Johns Hopkins University Press.

7 Rheinberger, for example, applies a similar framework to genetics. 
President's Commission for the Study of Ethical Problems in Medicine and Biomedical and Behavioral Research. 1981. Defining Death: A Report on the Medical, Legal and Ethical Issues in the Determination of Death. Washington D.C.: U.S. Government Printing Office.

Prusiner, Stanley. 1982. Novel Proteinasceous Infectious Particles Cause Scrapie. Science vol. 216 no. 4542: 136-44. doi:10.1126/science.6801762

Prusiner, Stanley, Michael P. McKinley, Darlene F. Groth, Karen A. Bowman, Nell I. Mock, S. Patricia Cochran, and Frank R Masiarz. 1981. Scrapie Agent Contains a Hydrophobic Protein. Proceedings of the National Academy of Sciences. Vol. 78 no. 11: 6675-97. doi:10.1073/pnas.78.11.6675

Reichenbach, Hans. 1958. The Philosophy of Space and Time. Trans. Maria Reichenbach. New York, NY: Dover. [Orig. pub. 1928. Philosophie der Raum-Zeit-Lehre. Berlin and Leipzig: Walter de Gruyter \& Co.]

Reichenbach, Hans. 1965. The Theory of Relativity and A Priori Knowledge. Trans. Maria Reichenbach. Berkeley, CA: University of California Press. [Orig. pub. 1920. Relativitäts-theorie und Erkenntnis Apriori. Berlin: Springer.]

Rheinberger, Hans-Jörg. 1997. Toward a History of Epistemic Things. Palo Alto, CA: Stanford University Press.

Stamp, J.T. 1962. Scrapie: A Transmissible Disease of Sheep. Veterinary Record vol. 74: 357-62.

van Fraassen, Bas C. 2008. Scientific Representation. New York, NY: Oxford University Press.

Whetstine, Leslie. 2008. The History of the Definition(s) of Death: From the 18th Century to the 20th Century. In End-of-Life Communication In The ICU: A Global Perspective, ed. David Cripen. 65-78. New York, NY: Springer Publishing.

Willis, R. 1878. William Harvey: A History of the Discovery of the Circulation of Blood. London, UK: C. Kegan Paul \& Co. Publishing. 